\title{
Characterization of the components present in the active fractions of health gingers (Curcuma xanthorrhiza and Zingiber zerumbet)by HPLC-DAD-ESIMS
}

\begin{abstract}
Curcuma xanthorrhiza and Zingiber zerumbet are two of the most commonly used ingredients in Indo-Malaysian traditional medicines, health supplements and tonics. Recently, a number of products derived from the aqueous extracts of these species have appeared in the market in the form of spray-dried powder packed in sachet or bottle. On-line high performance liquid chromatography, coupled with diode array detection and electrospray ion trap tandem mass spectroscopy (HPLC-DAD-ESI-MSn), was used to analyze the components in the antioxidant-active fractions from the rhizomes of these species. Three components were identified from C. xanthorrhiza, including bisdemethoxycurcumin (1), demethoxycurcumin (2) and curcumin (3). The active fraction from $\mathrm{Z}$. zerumbet consisted of five components, including kaempferol 3-O-rhamnoside (4), compound 5 [kaempferol 3-O-(200-Oacetyl)rhamnoside (5a) or kaempferol 3-O- (300-O-acetyl)rhamnoside (5b)], kaempferol 3-O(400-O-acetyl)rhamnoside (6), kaempferol 3-O-(300,400-O-diacetyl)rhamnoside (7) and kaempferol 3-O-(200,400-O-diacetyl)rhamnoside (8). To confirm their identities, the components from $\mathrm{Z}$. zerumbet were isolated conventionally and were analyzed by spectroscopic techniques as well as by comparison with literature data.
\end{abstract}

Keyword: Curcuma xanthorrhiza, Zingiber zerumbet, Traditional medicines, Health supplements, Tonics, Antioxidant activity, LC-DAD-ESIMS 